zu überwinden und klare, einfach nachzuvollziehende Teilhaberechte schafft, solange wirken Verunsicherung, Angst und das Gefühl, willkürlichen Entscheidungen ausgesetzt zu sein, der Wahrnehmung der bestehenden Möglichkeiten und Rechte entgegen.

Die angesichts der differenzierten Ausgestaltung erforderlichen Beratungsmöglichkeiten sind nicht verfügbar. Den Sozialbehörden gelingt es zunehmend weniger, ihren Beratungspflichten nach $\mathbb{S} \mathbb{S} 14,15$ SGB I nachzukommen. Eine unabhängige Beratung scheitert an viel zu wenig Anlaufstellen und auch an einer Überforderung vieler Berater(innen) angesichts der rechtlichen Komplexität. Solange diese weiter besteht, würde ich mir eine stärkere Kooperation der freien Wohlfahrtspflege mit der Anwältinnenschaft wünschen, die im Rahmen einer Betreuung nach $\mathbb{} 6$ Abs. 2 Rechtsdienstleistungsgesetz wichtige Funktionen übernehmen könnten. Dafür müssen dann aber auch die erforderlichen Gelder von der öffentlichen Hand bereitgestellt werden.

Der Einsatz für eine gleichberechtigte berufliche Teilhabe von Migrantinnen und die Anerkennung ihrer gesellschaftlich relevanten Leistungen außerhalb der Erwerbsarbeit sollte einen vollwertigen Platz in den rechtspolitischen Gleichstellungsdiskursen erhalten.

Zuzulassen, dass Migrantinnen systematisch in den Bereich unterbezahlter, prekärer persönlicher Dienstleistungen abgeschoben werden, wäre ein Verrat am Kampf der Frauenbewegung gegen die Zuweisung zu „Kinder, Küche, Kirche“.

\title{
Arbeitsmarktzugang von Migrantinnen und Migranten in der Praxis
}

\author{
Ein Erfahrungsbericht aus der Arbeit mit Migrant(inn)en in Projekten und Maßnahmen des Inter- \\ nationalen Bundes (e.V.) ${ }^{1}$
}

\author{
Anja Steuer-Loitsch \\ Programmgeschäftsführerin, Internationaler Bund, Heilbronn
}

\section{Arbeitswelt und Trends in Deutschland ${ }^{2}$}

Zentrale Thesen dieser interessanten Untersuchung sind:

- Deutschlands Bevölkerung schrumpft und wird immer älter.

- Die Bedeutung des lebenslangen Lernens steigt.

- Der Arbeitsmarkt verändert sich.

- Frauen haben zunehmende Chancen am Arbeitsmarkt.

- Der Arbeitsmarkt bietet vielfältige Möglichkeiten.

- Die Beschäftigungsprofile des Einzelnen verändert sich. Alle diese Faktoren betreffen die arbeitende Bevölkerung gleichermaßen, unabhängig von ihrer Herkunft. Da aufgrund der demografischen Entwicklung die Beschäftigungsquote von Migrant(inn)en jedoch steigen wird, sind diese Thesen im Bezug auf die Chancen und Perspektiven der Arbeitsmarktintegration dieser Bevölkerungsgruppe von besonderem Interesse. Der Bedeutungszuwachs von Wissen erhöht die Eintrittsbarriere am Arbeitsmarkt. Denn Unternehmen in Hochlohnländern sind vor allem auf ihre Innovationskraft und ihre Innovationsfähigkeit angewiesen. Auch der Umgang mit Komplexität und Veränderungsgeschwindigkeit ist für Arbeitnehmer(innen) eine sich weiter fortsetzende Herausforderung, der sie sich in ihrer Berufsbiografie stellen müssen. Konkret bedeutet dies, dass es solcher Mitarbeiter(innen) und Führungskräfte bedarf, die flexibel, mobil und anpassungsfähig sind und mit Unsicherheiten der Globalisierung (Dynamik, Schnelligkeit, Instabilität von Systemen, Unvorhersehbarkeit von Entwicklungen u.ä.m.) umgehen können.
Die Spaltung des Arbeitsmarktes (Fachkräfteengpass bei gleichzeitig hoher Arbeitslosigkeit) ist bereits heute deutlich spürbar und wird sich in Zukunft weiter verstärken. Dabei sind die Potenziale der Bevölkerung mit Migrationshintergrund gefragt.

Hier sieht das Referat für die Förderung von Integrationsprojekten für Zuwanderer ${ }^{3}$ eine Chance für Zuwander(innen) er, da diese im Zuge ihrer Integration bei uns die aus dem Herkunftsland mitgebrachten Qualifikationen, Kompetenzen und Fähigkeiten zur Anerkennung und Stärkung bringen können. Hieraus resultiert ein Ansatz des Bundesamtes für Migration und Flüchtlinge, der sogenannte „ressourcenorientierte Integrationsansatz“. Hierzu gibt es bereits zahlreiche Projekte, die die Talente und besonderen Fähigkeiten von $\mathrm{Zu}$ gewanderten aufgreifen und diesen somit zur Entfaltung verhelfen. In diesen Projekten geht es den Verantwortlichen darum, junge Frauen und Mädchen mit Migrationshintergrund zu ermutigen, die bisherigen Vorstellungen der Frauenrolle zu überdenken und die engen Grenzen nachbarschaftlicher sozialer Kontrolle zu überschreiten. Der Übergang in

1 Der Internationale Bund (IB) ist ein freier Träger der Jugend-, Sozialund Bildungsarbeit e.V. mit rund 8000 Mitarbeiter(inne)n an etwa 600 Standorten im Bundesgebiet, gegründet 1949 als Jugendsozialwerk: www.internationaler-bund.de.

2 Arbeit der Zukunft-Zukunft der Arbeit. Anforderungen und Herausforderungen. Ibe (Institut für Beschäftigung und Employability), Prof. Dr. Jutta Rump, April 2009

3 Martin, Andreas, Mitgebrachte Kompetenzen stärken, in: Bundesamt für Migration und Flüchtlinge (Hrsg.), Blickpunkt Integration 2 (02/2009), 9, erhältlich im Internet: <http://www.soziales.sachsen. de/download/OPEN_Workshop2_BAMF_Anerkennung auslaendischer_Bildungsabschluesse.pdf > (besucht am 11.1.2010). 
Ausbildung oder höhere Schulbildung soll geebnet werden, um den jungen Frauen stabile Perspektiven für eine selbstbestimmte Zukunft zu eröffnen. Auch die gesellschaftliche und politische Teilhabe wird vor diesem Hintergrund in den Praxisprojekten des Bundesamtes für Migration und Flüchtlinge gefördert.

Noch im Juli 2009 diskutierte der Landesarbeitskreis Integration (LAKI) Baden-Württemberg die Ausbildungssituation von Jugendlichen mit Migrationshintergrund und kam zu dem Schluss, dass „... eine fundierte Ausbildung der Garant für ein unabhängiges und selbstbestimmtes Leben, ist und „dass wir „auf die Potenziale der Jugendlichen mit Migrationshintergrund nicht verzichten dürfen". ${ }^{4}$ Denn eine fundierte Berufsausbildung ist eine entscheidende Voraussetzung für eine erfolgreiche Teilhabe am Arbeitsmarkt und somit auch am wirtschaftlichen und gesellschaftlichen Leben insgesamt - so konstatierten die Mitglieder des Arbeitskreises. Auch wiesen sie darauf hin, dass die noch nicht ausreichend genutzten Potenziale und Ressourcen der Migrant(inn)en wie die Mehrsprachigkeit und das kulturelle Wissen dieser Menschen Vorteile für Unternehmen in Zeiten der Globalisierung bedeuten können.

Doch ist die Ausbildungssituation der Jugendlichen mit Migrationshintergrund trotz vieler Programme und Maßnahmen noch nicht zufriedenstellend, da noch immer rund 32 Prozent der 25-bis unter 35-jährigen Migrant(inn)en über keinen beruflichen Abschluss verfügen. Bei Gleichaltrigen ohne Migrationshintergrund trifft dies lediglich auf circa acht Prozent zu.

Die Ursachen sieht der Landesarbeitskreis Integration vor allem in der teils noch unzureichenden schulischen Bildungssituation bei Migrant(inn)en, die den Übergang in Ausbildung deutlich erschwere. Fehlende Kenntnisse über das Schulsystem sowie das Ausbildungssystem sind ebenfalls ein Grund für diese Situation. Auch das sehr beschränkte Spektrum bei der Wahl des Ausbildungsberufs ist immer noch ein Thema ${ }^{5}$, sodass eine Kampagne zur Erweiterung des Berufswahlspektrums von Migrant(inn)en hier Abhilfe schaffen könnte. Eine Idee, die über die aktuellen Ausschreibungen des Europäischen Sozialfonds bereits aufgegriffen wird.

Parallel zur öffentlichen Diskussion über die Schwierigkeiten jugendlicher Migrant(inn)en an der Schwelle zur Ausbildung scheint es jedoch ebenso wichtig, die Verfahren zur Anerkennung von Schul- und Bildungsabschlüssen insgesamt zu vereinfachen und hier die erforderliche Transparenz zu schaffen.

Erfreulicherweise wird der Arbeitsmarkt der Zukunft eine höhere Teilhabe von Frauen mit sich bringen, eine Entwicklung, von der auch die Migrantinnen profitieren können. Dem zunehmenden Fachkräftebedarf auf dem Arbeitsmarkt wird durch die zunehmende Angleichung der Bildungsniveaus von Frauen Rechnung getragen. Damit einher geht eine Aufhebung tradierter Rollenverständnisse ebenso wie die steigende Bedeutung von Sozialkompetenzen in der Arbeitswelt. Frauen stehen dem Arbeitsmarkt auch deshalb in höherem Maße zur
Verfügung, da traditionelle Lebensentwürfe an Bedeutung verlieren. ${ }^{6}$

Die Anpassung der Einzelnen an die Anforderungen der Arbeitswelt wird zukünftig durch das „Profil der Beschäftigungsfähigkeit“ geprägt. Dieses Profil wird für alle Arbeitsbereiche, Berufsbilder und Hierarchie-Ebenen gelten und die gesamte Lebensarbeitszeit prägen. Dabei geht es um die Bereitschaft des Einzelnen, lebenslang Fachwissen aktuell bereit zu halten, lebenslang mit Vielfalt und Veränderung zurechtzukommen und mit Schlüsselkompetenzen den Anforderungen des Arbeitslebens zu begegnen. All dies erfordert ein hohes Maß an Eigenverantwortung und Eigeninitiative. ${ }^{7}$ Der Arbeitsmarkt der Zukunft verlangt also von seinen Akteur(inn)en zur Vermeidung beruflichen Abstiegs ein lebenslanges Training und Bereithalten von Fertigkeiten, Fähigkeiten und Kompetenzen. Der Arbeitsmarkt verlangt einen reibungslosen Übergang an der Schwelle Schule/Ausbildung, eine hohe Anzahl von Fachkräften und prognostiziert eine höhere Teilhabe von Frauen am Erwerbsleben.

\section{Arbeitsmarktintegration ausländischer Frauen - Ergebnisse einer vom Bundesamt durchgeführten Repräsentativunter- suchung ausgewählter Migrantengruppen ${ }^{8}$}

Wie viele Frauen aus der Türkei, Italien, Griechenland, Polen sowie den Nachfolgestaaten des ehemaligen Jugoslawien sind erwerbstätig und welche beruflichen Positionen haben sie inne? Diese für unser heutiges Thema interessante Untersuchung bezog sich auf die Zielgruppe der 18- bis unter 65-jährigen Frauen mit Migrationshintergrund; dabei wurden die fünf größten Ausländergruppen (ehemalige Anwerbeländer) zugrunde gelegt. Indikatoren der Untersuchung waren der Grad der Erwerbsbeteiligung sowie die Stellung im Beruf. Wichtigste Erkenntnisse dieser Repräsentativuntersuchung ausgewählter Migrantengruppen (RAM) sind, dass bei den untersuchten Frauen ein erhebliches Potenzial für den Arbeitsmarkt besteht und der Schlüssel zur Integration in den Arbeitsmarkt eine gute Ausbildung ist. Jedoch wurde auch herausgearbeitet, dass nur eine Minderheit der untersuchten Frauen ausländischer Staatsangehörigkeit im Alter zwischen 18 bis 65 Jahren gemessen an den Indikatoren „Erwerbsbeteiligung “ sowie „Stellung im Beruf“ gut in den Arbeitsmarkt integriert ist und einer qualifizierten oder hochqualifizierten Tätigkeit nachgeht. Der Anteil der beruflich gut positionierten Frauen beträgt 16 Prozent. Erhebliche Unterschiede zwischen den Herkunftsgruppen und insbesondere den Generationen konnten herausgearbeitet werden:

4 Medieninformation des LAKI vom 13.07.2009, Zitat von Justizminister Prof. Dr. Ulrich Goll, Integrationsbeauftragter des Landes Baden-Württemberg. Der LAKI setzt sich u.a. aus Vertretern und Vertreterinnen aus Politik, Verbänden, der Wohlfahrtspflege, den Kirchen sowie den kommunalen Migrantenvertretungen des Landes zusammen.

5 Prof. Dr. Goll, ebd.

6 Prof. Dr. J. Rump, Arbeit der Zukunft - Zukunft der Arbeit, ibe, 04 2009, 10.

7 Ebd., $16 \mathrm{f}$. 
Bei den Angehörigen der ersten Generation bewegt sich die Quote gut positionierter Frauen zwischen drei Prozent bei den Türkinnen und 22 Prozent bei den Polinnen.

Bei den Angehörigen der zweiten Generation ist der niedrigste Anteil ebenfalls bei den Türkinnen zu finden, von denen 22 Prozent eine qualifizierte bis hochqualifizierte Tätigkeit ausüben.

Bei den in Deutschland aufgewachsenen Griechinnen beläuft sich der entsprechende Anteil auf 53 Prozent.

Durch die Untersuchung konnte herausgearbeitet werden, dass die Arbeitsmarktintegration sowohl durch sozialstrukturelle Merkmale wie Alter, Generationszugehörigkeit, familiäre Situation als auch durch erworbene Qualifikationen der Frauen, so etwa Deutschkenntnisse, schulische und berufliche Bildung beeinflusst wird. ${ }^{9}$ Ein weiterer wichtiger Befund besteht darin, dass die Wahrscheinlichkeit, erwerbstätig zu sein, bei allen Herkunftsgruppen - mit Ausnahme der Türkinnen - weniger durch das Herkunftsland als durch die Altersklasse, der sie angehören, die Aufenthaltsdauer, die Generationenzugehörigkeit, den Familienstand, die Zahl und das Alter der Kinder sowie das Zuwanderungsmotiv bestimmt ist. Interessant ist schließlich die Frage, wie sich neben den sozialstrukturellen Merkmalen die erworbenen Qualifikationen, nämlich Deutschkenntnisse sowie formale Schul- und Berufsabschlüsse, auf die Beschäftigungsquote auswirken: Frauen, die ihrer Einschätzung nach gut oder sehr gut deutsch sprechen, die über einen Schulabschluss oder über eine Berufsausbildung verfügen, sind anteilsmäßig häufiger erwerbstätig als Frauen mit schlechten Deutschkenntnissen, als Frauen ohne Schulabschluss (oder mit schlechtem Schulabschluss) oder ohne Berufsausbildung.

Bezogen auf die Schul- und Ausbildungsabschlüsse bestätigte sich außerdem, dass es nicht nur auf die Höhe des jeweiligen Abschlusses ankommt, sondern auch darauf, ob dieser in Deutschland anerkannt wird! Insgesamt verdeutlicht die Untersuchung, dass sowohl die Schulbildung als auch der berufliche Ausbildungsstand von Bedeutung für die Erwerbstätigkeit aller fünf Herkunftsgruppen ist. Nicht nur die Beschäftigungsquote nimmt mit der Höhe der beruflichen Qualifikation zu, sondern auch die Quote der Ausübung einer qualifizierten oder hochqualifizierten Tätigkeit. Gleichzeitig wird deutlich, dass bei diesen Frauen ein erheblicher Nachqualifizierungsbedarf besteht, dies insbesondere für Migrantinnen der ersten Generation, von denen zwei Drittel keinen oder keinen in Deutschland anerkannten Berufsabschluss vorweisen können. Insgesamt wird so konstatiert, dass im Bezug auf Migrantinnen Programme zur Förderung der Arbeitsmarktintegration notwendig sind. Bedeutsam zur besseren Arbeitsmarktintegration bereits ausgebildeter Migrantinnen der ersten Generation ist es, mehr Angebote zu schaffen, die es den Frauen ermöglichen, ihre aus den Herkunftsländern mitgebrachten Qualifikationen in Deutschland zu verwerten, sei es durch die Anerkennung vergleichbarer Ausbildungsabschlüsse, sei es durch Nachschulungsmöglichkeiten, bei denen die vorhandenen Berufskenntnisse an die Erfordernisse des deutschen Arbeits- marktes angepasst werden. Um auch die Arbeitsmarktchancen der zahlreichen Migrantinnen ohne Berufsausbildung sowohl der ersten als auch der zweiten Generation zu verbessern, kommt nicht zuletzt dem Ausbau von Maßnahmen zum Nachholen berufsqualifizierender Abschlüsse eine hohe Bedeutung zu. Hierbei scheint es sinnvoll, gezielt Angebote für Mütter zu schaffen.

\section{Praxisbeispiele für die Arbeitsmarktintegration von Migrant(inn)en}

\section{Praxisbeispiel Jugendmigrationsdienste}

Erste Anlaufstelle vieler Zuwander(innen)er in Deutschland sind die Jugendmigrationsdienste: vor über 50 Jahren wurde der erste (damals noch Jugendgemeinschaftswerk) im Internationalen Bund (IB) gegründet, seither sind rund 100 Standorte in Deutschland dazugekommen. ${ }^{10}$ Jugendmigrationsdienste gibt es bundesweit in der Trägerschaft der Kirchen und Wohlfahrtsverbände sowie in freier Trägerschaft. Bis vor einigen Jahren kümmerten sich die Jugendgemeinschaftswerke insbesondere um junge Spätaussiedler, hauptsächlich aus den Staaten der ehemaligen Sowjetunion; dies geschah im Auftrag des Bundesministeriums für Familie, Senioren, Frauen und Jugend (BMFSFJ). Oft wurden und werden die jungen Leute mitten im problematischen Lebensalter an der Schwelle zum Erwachsenwerden aus ihrem alten Freundes- und Kulturkreis herausgerissen und müssen in Deutschland ein neues Leben anfangen. Viele dieser jungen Aussiedler sprechen kaum noch deutsch, obwohl das doch die beste Voraussetzung für eine erfolgreiche Integration in die neue Heimat wäre. An dieser Stelle nun bieten die Jugendmigrationsdienste ihre Hilfe an. Sie beraten und unterstützen beim Erwerb der deutschen Sprache, bei der Entscheidung über die Schullaufbahn, beim Versuch, neue Freunde zu finden und natürlich bei der Suche eines Ausbildungs- oder Arbeitsplatzes. Jedoch stehen viele andere Jugendliche vor den gleichen Problemen wie die Spätaussiedler: Das sind die Jugendlichen nicht-deutscher Herkunft, d.h. solche, die neu zuwandern, oder solche, die zwar hier aufgewachsen sind, aber deren Familien nicht aus Deutschland stammen. Auch sie haben häufig aufgrund schlechter Deutschkenntnisse und niedrigerer Bildungsabschlüsse geringe Chancen auf dem Ausbildungs- und Arbeitsmarkt. Auch die Schwierigkeiten dieser Jugendlichen resultieren häufig aus der Erfahrung der Migration von einer bekannten Kultur und Gesellschaft in eine fremde - die deutsche. Das BMFSFJ hat daher vor drei Jahren den Jugendgemeinschaftswerken den Auftrag gegeben, alle jungen Menschen mit Migrationshintergrund im Alter von 12 bis 27

\footnotetext{
8 Arbeitsmarktintegration von Frauen ausländischer Nationalität in Deutschland, Publikationen des Bundesamtes für Migration und Flüchtlinge, Working Paper 20, 2008.

9 Ebd., 51.

10 Der Internationale Bund e.V. betreibt bundesweit 65 Jugendmigrationsdienste mit 36 Außenstellen für 20.00o Zuwanderer (vgl. Geschäftsbericht der IB-Gruppe 2007/2008).
} 
Jahren bei ihrem Integrationsprozess in die deutsche Gesellschaft zu unterstützen.

Alle Neuzuwanderer, auch die nicht mehr Schulpflichtigen zwischen 16 und 27 Jahren, müssen innerhalb einer bestimmten Zeit nach ihrer Ankunft in Deutschland einen Integrationskurs absolvieren, der aus einem Deutsch- und einem Orientierungskurs besteht. Hauptaufgabe der Jugendmigrationsdienste ist die sozialpädagogische Begleitung und individuelle Beratung der Jugendlichen während ihres Integrationsprozesses. Der (die) Mitarbeiter(in) ist somit Casemanager(in) der Jugendlichen und ermittelt mit diesen zunächst die persönlichen, schulischen oder beruflichen Voraussetzungen, die sie aus dem Herkunftsland mitbringen, um dann eine Vereinbarung über den weiteren Weg in Deutschland zu entwickeln. Der Weg in den Beruf kann beispielsweise über ein vom Jugendmigrationsdienst vermitteltes Praktikum oder in einen berufsorientierten Deutschkurs erfolgen. Ergänzend dazu unterstützen Gruppenangebote den Integrationsprozess; sie bieten unter anderem Orientierungshilfen im Ausbildungs- und Bildungssystem, vermitteln Computerkenntnisse oder fördern den Spracherwerb. Die Neuausrichtung der Jugendmigrationsdienste impliziert auch die Wahrnehmung von sozialräumlichen Vernetzungsaufgaben im kommunalen und regionalen Einzugsbereich. Betriebe, Schulen, Beratungsstellen, Jugend- und Sozialämter, die örtlichen Arbeitsagenturen, Träger der Jugendberufshilfe gehören zum Netzwerk und arbeiten gemeinsam am Ziel der erfolgreichen Integration der Teilnehmenden.

Die individuelle Integrationsförderung hat also folgende Schwerpunkte:

- Aufnahme-/Beratungsgespräche

- Casemanagement

- Integrationskurse

- Berufsorientierte Deutsch- und Orientierungskurse

- Sozialräumliche Vernetzung

- Vermittlung interkultureller Kompetenzen

- Berufswegeplanung

- Unterstützung bei der Ausbildungs- oder Arbeitsplatzsuche

\section{Praxisbeispiel Integrationsprojekt}

Ein aktuelles Beispiel des IB in Berlin-Neukölln in Kooperation mit dem Diakonischen Werk ist das Integrationsprojekt JuMiLo. JuMiLo bedeutet: „Junge Migranten als Lotsen“. Diese Lots(inn)en sind vor einigen Jahren aus aller Herren Länder nach Deutschland eingereist und leben in verschiedenen Städten im Bundesgebiet. Die Lots(inn)en möchten künftig vor allem Gleichaltrige dabei unterstützen, in Deutschland Fuß zu fassen, etwa durch Patenschaften, Sprachfördergruppen, Nachhilfeunterricht und Bewerbungstraining. Das Projekt beginnt in diesem Jahr in 15 Jugendmigrationsdiensten deutschlandweit und ist auf drei Jahre angelegt. Gefördert wird JuMiLo aus Mitteln des Bundes und des Europäischen Sozialfonds. Die Lots(inn)en arbeiten ehrenamtlich und tragen somit ihren Teil zur Forderung des Bundes nach bürgerschaftlichem Engagement bei. Sie haben Vorbildfunktion und sollen andere ermutigen, es ihnen gleichzutun. Die Glaubwürdigkeit der Lots(inn)en ist daher so hoch, weil sie selbst aus ihrem eigenen Erleben berichten können und wissen, wie schwierig es ist, sich in Deutschland durchzusetzen.

\section{Schlussfolgerungen und Handlungsperspektiven}

\section{Vor dem Start in die berufliche Ausbildung steht die schulische Bildungslaufbahn}

Expert(inn)en sind der Meinung, dass die Verwirklichung von Chancengleichheit im Bildungssystem noch nicht der Realität entspricht. ${ }^{11}$ So sollen zwar alle Kinder und Jugendlichen unabhängig von ihrer sozialen Herkunft und ethnischen Abstammung an Bildung teilhaben können, jedoch setzt sich der Trend zur Ungleichheit und ungerecht verteilten Chancen fort.

\section{Am Ende der schulischen Bildungslaufbahn steht der Übergang in das System der beruflichen Bildung}

Berufliche Ausbildung ist die Voraussetzung für eine berufliche Integration und gesellschaftliche Teilhabe. Bedingt durch den Mangel an Ausbildungsplätzen in den letzten Jahren hat die Zeit des Übergangs von der Schule in den Beruf für viele junge Menschen den Charakter einer eigenständigen Lebensphase angenommen. Auch wenn alle Jugendlichen von diesem schwierigen Übergang betroffen sind, haben Jugendliche mit Migrationshintergrund die meisten Hindernisse zu überwinden, selbst bei gleichen schulischen Voraussetzungen.

\section{Neben der Integrationsgerechtigkeit des Schulsystems wird die Integrationsgerechtigkeit des Ausbildungssystems in Frage ge- stellt ${ }^{12}$}

Angesichts der erklärten bildungspolitischen Zielsetzung der Bundesregierung und der Sozialpartner, allen jungen Menschen in Deutschland eine Berufsausbildung zu ermöglichen, gilt es erheblich stärker als bisher, den Zugang junger Menschen mit Migrationshintergrund zu einer vollqualifizierenden Ausbildung zu fördern. Die Angebotsstrukturen sollen sich deutlich stärker an den jungen Migrant(inn)en orientieren, die trotz guter schulischer Voraussetzungen keinen Ausbildungsplatz finden.

\section{Eine differenziertere Betrachtungsweise von Ausbildungsplatz- bewerber(inne)n mit und ohne Migrationshintergrund scheint erforderlich}

Um allen jungen Menschen in Deutschland eine qualifizierende Berufsausbildung zu ermöglichen, ist eine breit angelegte und abgestimmte Offensive in einem integrierten Förderprogramm notwendig. Die Heterogenität der Schulabsolvent(inn)

11 News der Bundesarbeitsgemeinschaft Jugendsozialarbeit vom 22.9.2009 mit Hinweis auf den aktuellen WISO-Diskurs der Friedrich-Ebert-Stiftung.

12 Vgl. Beicht, Ursula/Granato, Mona, Übergänge in eine berufliche Ausbildung. Geringere Chancen und schwierige Wege für junge Menschen mit Migrationshintergrund - Expertise des Gesprächskreises Integration und Migration der Friedrich-Ebert-Stiftung, in: Friedrich-Ebert-Stiftung (Hrsg.): WISO Diskurs, Bonn 2009, 47. 
en sowie die der Altbewerber(innen) (mit und ohne Migrationshintergrund) im Hinblick auf Lernvoraussetzungen, Bildungsabschlüsse, Erstsprache, Alter und Geschlecht sollten bei der Umsetzung von Förderangeboten berücksichtigt werden.

\section{Die unterschiedlichen Handlungsfelder müssen miteinander korrespondieren}

Eine qualitative Verbesserung und eine quantitative Ausweitung präventiver Maßnahmen in der Berufsorientierung, die frühzeitig einsetzen, ist wünschenswert; ebenso wäre die zielgruppenspezifische Differenzierung in der Ausbildungsvorbereitung bis hin zur Verbesserung der Zugänge und Durchführung vollqualifizierender Ausbildung (betrieblich oder außerbetrieblich) in den Blick zu nehmen. Außerdem sollte die abschlussbezogene Nachqualifizierung junger Erwachsener - mit und ohne Migrationshintergrund - als Bestandteil eines integrierten Förderprogramms mit berücksichtigt werden.

\section{Das Maßnahmebündel sollte regional gesteuert und umge- setzt werden \\ Die bisher praktizierte quantitative Ausweitung der Bildungs- angebote im Übergang Schule/Beruf hat vor Ort zu einer er- heblichen Intransparenz der Angebote geführt. Idealerweise könnte ein regionales Übergangsmanagement dazu beitragen, den Förderbedarf zu koordinieren; die bisher vorhandenen Förderangebote könnten systematisiert werden, die regionalen Akteur(inn)e(n) zusammengebracht und vernetzt werden.}

Vielfalt als Chance sehen, interkulturelle Fähigkeiten nutzen Der Weg zu einer breiten Nutzung interkultureller Fähigkeiten führt über die Sensibilisierung dafür, d.h. ihre Wahrnehmung,
Förderung und Wertschätzung durch die Unternehmen. Interkulturelle Kompetenzen müssen sichtbar gemacht werden. Unabdingbar ist die dauerhafte Verankerung der Schlüsselqualifikation „interkulturelle Kompetenz“ im gesamten Umfeld beruflicher Ausbildung, also für Ausbildungspersonal, Berufsschullehrer(innen), Körperschaften.

\section{Mentoring ausbauen}

Die Begleitung durch Pat(inn)en mit Vorbildfunktion durch den gesamten Orientierungs-, Übergangs- und Qualifizierungsprozess hindurch bis zu einer stabilen Einmündung in den Beruf könnte erheblich zum Gelingen der Qualifizierung und Einmündung in den Beruf beitragen. Mentor(inn)en könnten in ihrer Funktion als Brückenbauer als Ansprechpartner(innen) für Unternehmen und Netzwerke fungieren.

\section{Fazit}

Das Ziel einer gleichberechtigten Teilhabe von Migrant(inn)en - aber auch von sogenannten benachteiligten Gruppen - kann letztlich nur durch eine gleichberechtigte Teilhabe an Bildung erreicht werden. Bildungsgerechtigkeit ist die Voraussetzung für das Erreichen von Chancengerechtigkeit im Ausbildungssystem. Ohne qualifizierte Ausbildung ist Partizipation am Arbeitsmarkt von vornherein nur eingeschränkt möglich. Daher ist es auch wichtig, die Verwertung bereits erzielter Abschlüsse durch Anerkennung oder gegebenenfalls Anpassungsqualifizierung im höheren Maße als bisher zu ermöglichen. Der Arbeitsmarkt sollte - vor dem Hintergrund seiner modernen Anforderungen an alle - unabhängig von sozialer und ethnischer Herkunft zugänglich sein.

\section{Gesundheit und soziale Teilhabe Qualität von und Gleichheit beim Zugang zu Gesundheitsdienstleistungen}

\section{Dr. Annette Matthias}

Hauptverwaltungsrätin bei der Europäischen Kommission in Brüssel, GD Wettbewerb

\footnotetext{
Hintergrund

Gleicher Zugang für alle zu einer Gesundheitsversorgung von hoher Qualität - dies ist eine Kernherausforderung der Gesundheitssysteme in ganz Europa. Trotz bestehender Unterschiede in Größe, Struktur und Finanzierung der Gesundheitssysteme zeigt uns die Realität, dass europaweit bestimmte Bevölkerungsgruppen in unverhältnismäßiger Weise auf Hindernisse beim Zugang zur Gesundheitsversorgung stoßen. Studien haben gezeigt, dass der Zugang zur Gesundheitsversorgung mit Armut und sozialer Ausgrenzung abnimmt.
}

Auch auf EU-Ebene ist der Zugang zur Gesundheitsversorgung eine Kernfrage. Der Zugang für alle zu einer angemessenen Gesundheitsversorgung und Langzeitpflege sowie die Beseitigung der Ungleichheiten beim Zugang zu einer solchen Gesundheitsversorgung wurde vom Rat der Europäischen Union im Juni 2006 als ein prioritäres Ziel der neuen gestrafften Offenen Methode der Koordinierung der EU für Sozialschutz und soziale Eingliederung vorgeschlagen. Die von der Europäischen Kommission im Jahr 2006 vorgelegte Strategie für die soziale Eingliederung legt darüber hinaus den Hauptschwerpunkt auf die Verringerung der Armut und der sozialen Ausgrenzung der EU-Bürger(innen).

Vor diesem Hintergrund hat die Europäische Kommission (GD Beschäftigung, soziale Angelegenheiten und Chancengleichheit) die European Health Management Association mit 